\title{
Coordinación entre docencia e investigación, para la mejora de la comprensión de procesos y la motivación de los estudiantes de ingeniería, y la colaboración entre docentes, para reducir el esfuerzo en innovación
}

\author{
Naves García-Rendueles, Juan'; Naves García-Rendueles, Acacia²; Anta Álvarez, Jose ${ }^{1}$ \\ ${ }^{1}$ Universidade da Coruña, Grupo de Ingeniería del Agua y del Medio Ambiente, Escuela \\ Técnica Superior de Caminos, Canales y Puertos \\ ${ }^{2}$ Universidade da Coruña, Centro de Investigaciones Científicas Avanzadas (CICA), Escuela \\ Técnica Superior de Caminos, Canales y Puertos
}

\section{RESUMEN}

En este trabajo se presenta una experiencia de coordinación entre los docentes de dos asignaturas de Ios Másters de Ingeniería de Caminos y de Ingeniería del Agua de la Universidade da Coruña para, a través del empleo de experiencias de investigación, fomentar la motivación y los resultados del aprendizaje en la enseñanza del drenaje urbano. Dentro de las actividades docentes se realiza una visita a una instalación experimental en el Centro de Innovación Tecnológica en Edificación e Ingeniería Civil que consiste en un simulador de lluvia con calles, tejados y una red de drenaje completa. Una vez realizada la visita, los estudiantes realizan un modelo numérico con software profesional de dicha instalación. La visita al laboratorio, con la observación de los procesos in situ, y la aplicación del software a un entorno conocido, consiguen mejorar la compresión de los contenidos de las materias y motivar al alumnado.

PALABRAS CLAVE: coordinación docente, drenaje urbano, uso de software, investigación 


\section{CITA RECOMENDADA:}

Naves García-Rendueles, J.; Naves García-Rendueles, A.; Anta Álvarez, J. (2019): Coordinación entre docencia e investigación, para la mejora de la comprensión de procesos y la motivación de los estudiantes de ingeniería, y la colaboración entre docentes, para reducir el esfuerzo en innovación. En De la Torre Fernández, E. (ed.) (2019). Contextos universitarios transformadores: construíndo espazos de aprendizaxe. III Xornadas de Innovación Docente. Cufie. Universidade da Coruña. A Coruña (pág. 469-478).

DOl capítulo: https://doi.org/10.17979/spudc.9788497497121.469

DOl libro: https://doi.org/10.17979/spudc.9788497497121

\section{ABSTRACT}

A coordinated activity between the lecturers of two subjects of Master of Civil Enginering and the International Master of Water Engineering of the University of A Coruña is here presented. The main goal is promoting the motivation and learning outcomes in the teaching of urban drainage by means of research activities. First, a technical visit is performed to a rainfall simulator with roads and roofs placed at the Center for Technological Innovation in Construction and Civil Engineering. After the visit, the students develop a professional simulation model of this facility using a technical software. The visit, including the observation of the processes in situ, and the development of the numerical modelling to a well-known and actual environment, allows to improve the comprehension of the contents of the subjects and the motivation of the students.

KEY WORDS: teaching coordination, urban drainage, software use, research. 


\section{INTRODUCCIÓN}

Una de las competencias de los egresados en las titulaciones de Máster de Ingeniería de Caminos, Canales y Puertos y del International Master in Water Engineering de la Universidade da Coruña es el diseño de sistemas de saneamiento y drenaje urbano. En el ámbito del drenaje urbano, uno de los problemas que se deben resolver es el de evitar las inundaciones causadas por las lluvias torrenciales.

La docencia de esta temática se realiza en las asignaturas de Obras Hidráulicas e Hidrología y Water Supply and Drainage Systems, en los Másters de Ingeniería de Caminos y Water Engineering, respectivamente. Hasta hace pocos años, el contenido de las dos materias en el ámbito del drenaje urbano no estaba coordinado, y la metodología docente se basaba fundamentalmente en las clases magistrales, junto con la realización de una práctica a través de TICs empleando el software profesional de diseño de sistemas de drenaje Storm Water Management Model (SWWM) de la US-EPA (Rossman, 2015).

Con esta metodología no se conseguía la participación del alumnado en las clases y además la comprensión de los procesos involucrados en el estudio del drenaje urbano tenía cierta dificultad. Este es el caso de los procesos de transformación de lluvia en escorrentía o el movimiento del agua en las cuencas urbanas durante los episodios de precipitación. Además, debido al tiempo limitado para impartir cada bloque de las asignaturas, se presentaban ejemplos y prácticas simplificadas, que no favorecían que el estudiantado adquiriese una visión global de la problemática a analizar.

Con motivo de la primera convocatoria de Grupos de Innovación Educativa (GIE) de la UDC un grupo de profesores de distintas áreas de conocimiento con docencia en la Escuela de Caminos, Canales y Puertos creó el GIE Tecnologías Activas. Dentro de las actividades del grupo se desarrolló una experiencia para mejorar la docencia del drenaje urbano caracterizada por (i) involucrar la coordinación entre docentes de distintos masters, (ii) coordinar actividades de docencia e investigación y (iii) acercar la realidad física de la materia para motivar al alumnado y mostrarles la utilidad de los contenidos que se explican en clase. 


\section{DESCRICIÓN DE LA EXPERIENCIA}

En primer lugar, se coordinaron los contenidos de la docencia impartida en las asignaturas Obras Hidráulicas y Water Supply and Drainage Systems del Máster de Ingeniería de Caminos y de Ingeniería del Agua. La docencia se estructuró con las siguientes actividades:

- Clases magistrales. Se redujo el número de clases magistrales liberando tiempo para el resto de actividades de la materia.

- Clases prácticas. Se comenzaron a realizar un mayor número ejercicios prácticos en clase empleando las Instrucciones Técnicas para Obras Hidráulicas en Galicia (ITOHG), que son las referencias normativas sectoriales en el ámbito gallego (Augas de Galicia, 2009).

- Visitas a laboratorio. Se incluyó una visita al laboratorio de hidráulica del Centro de Innovación Tecnológica en Edificación e Ingeniería Civil (CITEEC) donde se cuenta con un simulador de lluvia.

- Prácticas con TICS. Se modificaron los trabajos realizados con el programa profesional SWMM, comenzando con la modelización de un ensayo de drenaje en realizado con el simulador de lluvia en las instalaciones visitadas.

Con respecto a la coordinación de actividades docentes y de investigación, se programó una visita al laboratorio de hidráulica del CITEEC de ambos grupos de alumnos y alumnas. Las instalaciones del laboratorio incluyen dos simuladores de lluvia diferentes. El primero de ellos es un simulador sobre un modelo físico de una calle, de unos $36 \mathrm{~m}^{2}$ de superficie (Figura 1). El modelo cuenta con la calzada, una acera, dos sumideros para la captación de la escorrentía y una red de drenaje compuesta por tres conducciones de $90 \mathrm{~mm}$ y $150 \mathrm{~mm}$. El modelo físico del segundo simulador (Figura 2) tiene una superficie aproximada de $100 \mathrm{~m}^{2}$ (escala de barrio). Incluye 2 calles, 6 bloques de edificios, 4 imbornales y 4 pozos de registro, así como una red de drenaje compuesta por tres conducciones de $200 \mathrm{~mm}$. Las conducciones de la red son transparentes y accesibles por la parte inferior del modelo físico. 


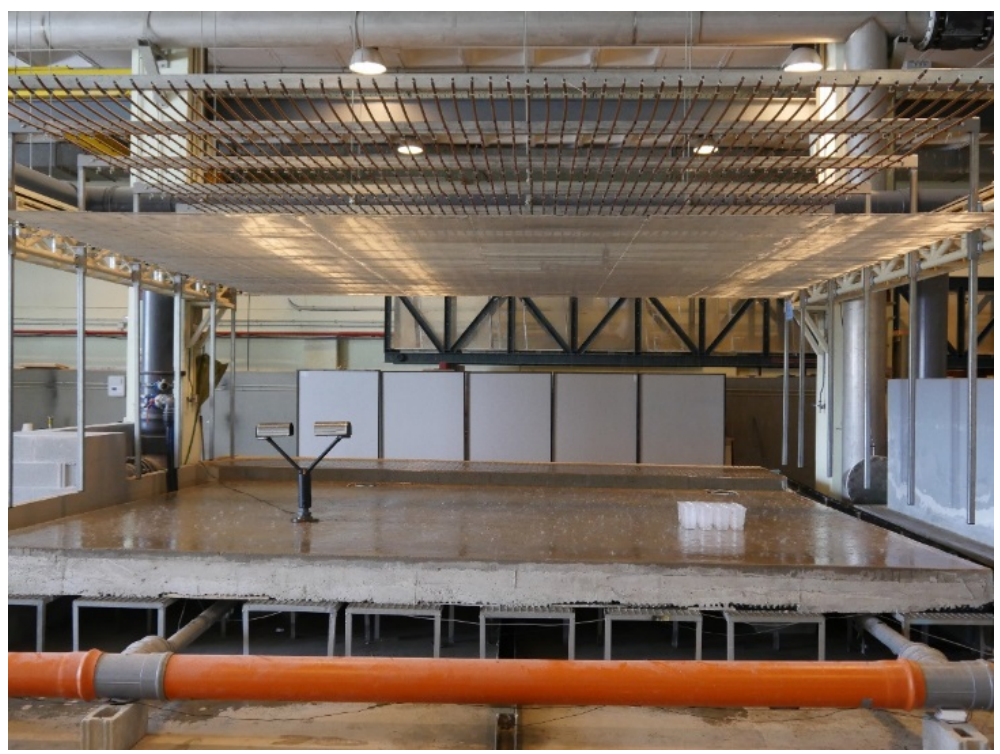

Figura 1. Modelo físico con simulador de lluvia a escala de calle en el laboratorio de hidráulica del CITEEC.

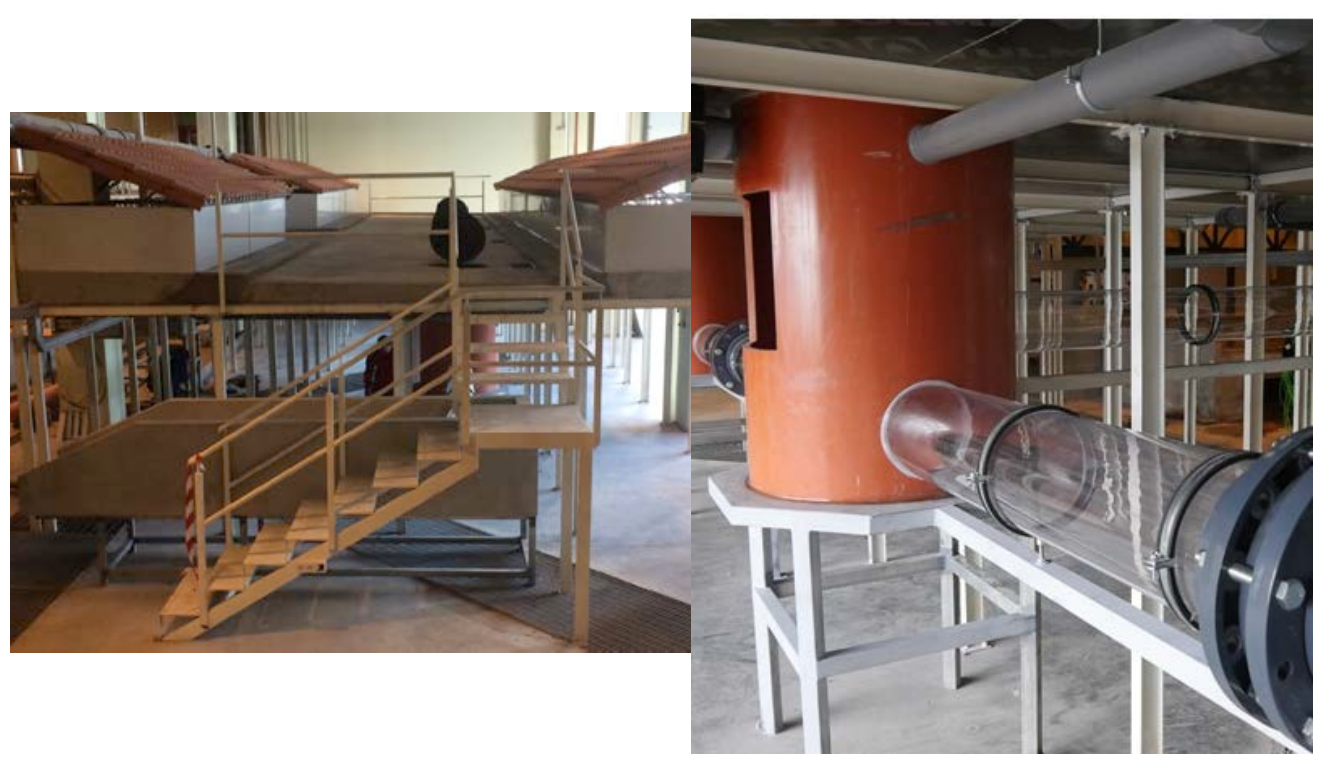

Figura 2. Modelo físico con simulador de lluvia a escala de barrio en el laboratorio de hidráulica del CITEEC. 
Durante la visita a las instalaciones del alumnado, se realiza una introducción al trabajo experimental desarrollado por los investigadores de la UDC en ellas. Se facilita información relativa a los simuladores y a los fundamentos de los equipos empleados en los ensayos de laboratorio para determinar los niveles de agua en la superficie 0 en las conducciones, la velocidad del flujo en la superficie de las calles 0 el caudal que circula por las tuberías. También se aborda el estudio de los procesos de generación y transporte de contaminación en los sistemas de drenaje urbano, mediante un pequeño ejemplo de transporte de un trazador en uno de los modelos físicos y la presentación de resultados recientes de investigación.

Por último, se realiza en clase con el alumnado una práctica que consiste en la simulación numérica del drenaje en uno de los modelos físicos visitados con el SWMM. Con esta actividad se pretende acercar a los alumnos la realidad física y mostrar las posibles aplicaciones en su futura vida profesional. El esquema conceptual del modelo (Figura 3) es lo suficientemente complejo para introducir los elementos más importantes que debe incluir un modelo numérico de un drenaje urbano: la geometría de las calles y tejados, la forma y disposición de las conducciones, la definición de los criterios para introducir las precipitaciones de diseño o los caudales circulantes de aguas residuales durante las condiciones de tiempo seco (sin lluvia). Para el desarrollo de esta práctica, se realizó una guía de resolución adaptada para cada una de las materias (una en castellano y otra en inglés). 


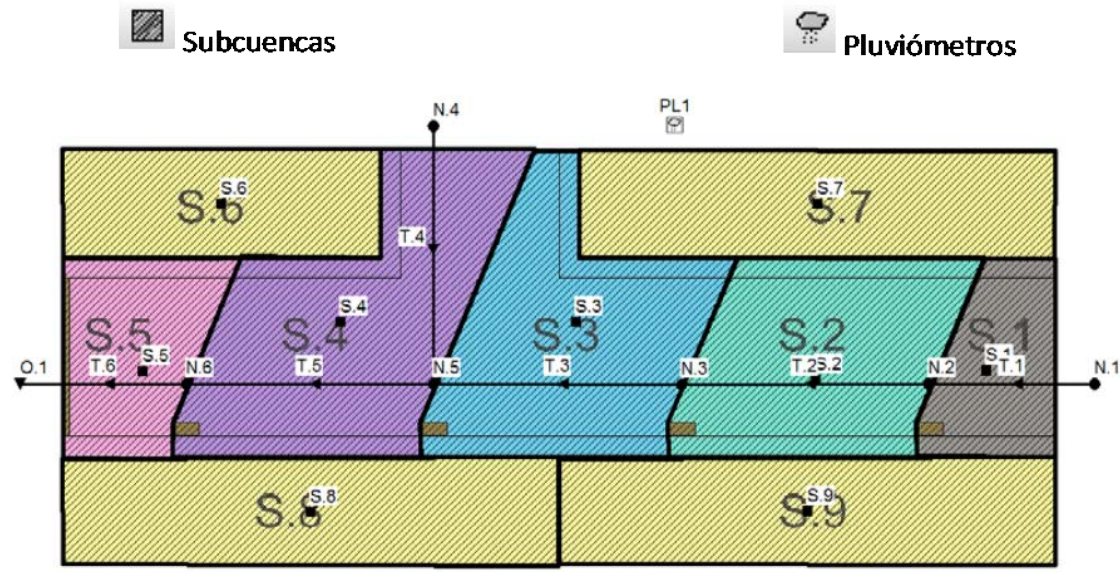

Figura 3. Esquema del modelo numérico realizado con SWMM del modelo físico a escala barrio del simulador de lluvia.

Una vez realizada esta práctica, se evalúan los conocimientos adquiridos de modelización numérica con la construcción de un modelo numérico de una cuenca de saneamiento real. Este modelo permite introducir el diseño de estructuras más complejas para la protección frente a inundaciones y el control de la contaminación.

\section{RESULTADOS}

La experiencia descrita en esta comunicación comenzó a desarrollarse en el curso 2017-18, continuó durante el curso 2018-19 y se pretende seguir con ella en próximos cursos.

Las visitas de los estudiantes a las instalaciones se realizaron con la asistencia de los profesores de las materias y de un estudiante de doctorado. La participación del estudiante de doctorado fue muy positiva, porque que cuenta con una edad más cercana a los alumnos y consiguió despertar el interés por el trabajo de investigación que se desarrollaba en las instalaciones visitadas.

La observación de los procesos de generación de escorrentía y movilización de contaminantes (Figura 4), facilitó y reforzó su comprensión. Además, durante el desarrollo de las visitas se 
generó un ambiente distendido en el que la participación del alumnado creció notablemente y favoreció un mejor ambiente en el aula en las siguientes sesiones.
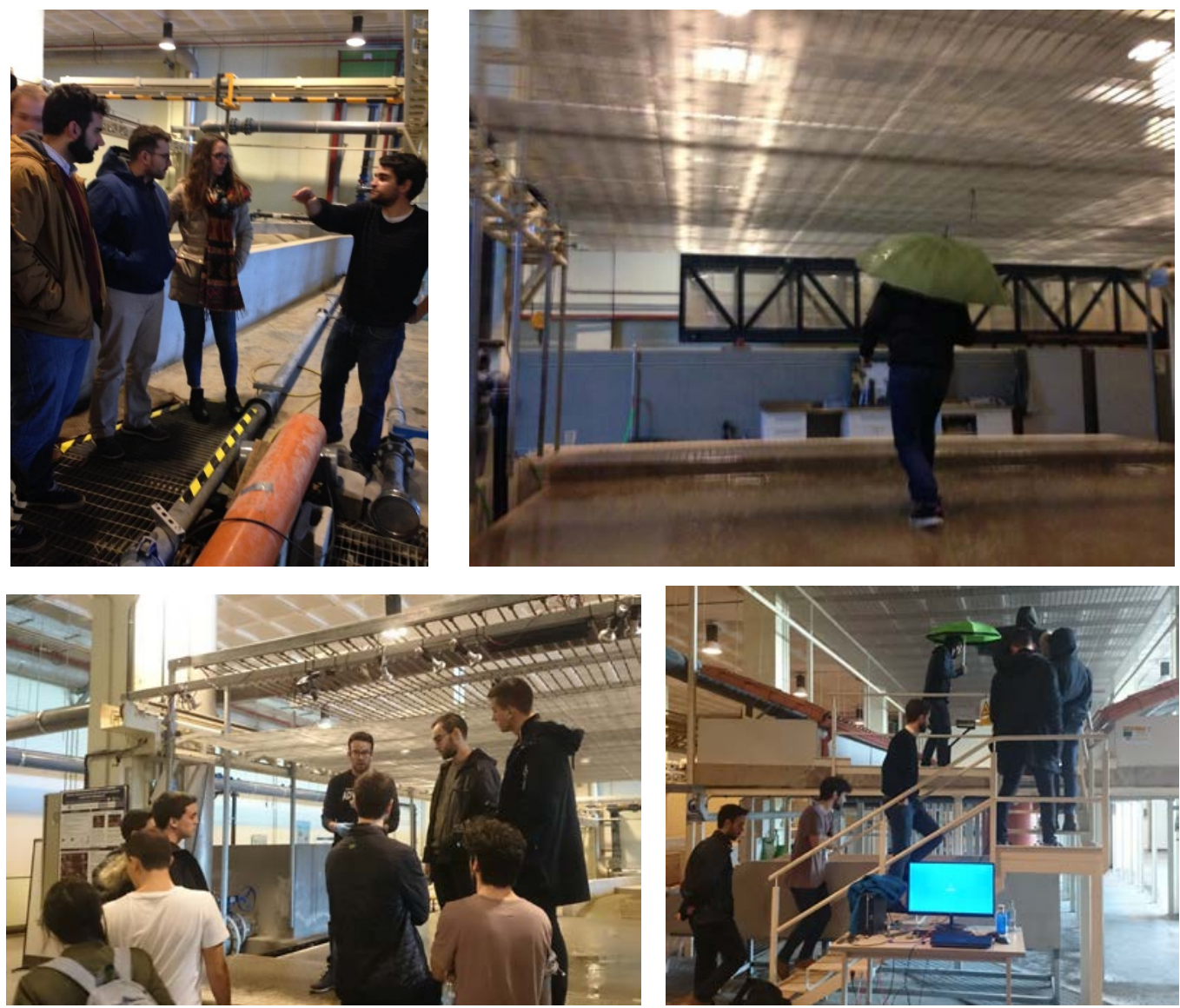

Figura 4. Fotografías de las visitas a los simuladores de lluvia.

Con la realización en clase del modelo numérico del modelo físico que los alumnos acababan de ver en el CITEEC, se consiguió reforzar los conceptos trabajados en la visita, familiarizar a los estudiantes el uso del software técnico (SWMM) y fomentar un análisis crítico de los resultados, al conocer el resultado real en el laboratorio. Por otro lado, la modelización de un 
sistema real con software profesional demostró al alumnado la utilidad de los conocimientos que se imparten y aprenden en clase.

El interés de los estudiantes y su satisfacción fueron evidentes tanto por su actitud como por la gran cantidad de preguntas que realizaron. Por otra parte, el profesorado también considera que la experiencia está siendo muy positiva en cuanto al incremento de la comprensión de los procesos y al interés del alumnado en la materia. Por último, la coordinación entre profesores de distintas titulaciones permitió ahorrar esfuerzos en la organización de la actividad, en la elaboración de material para clases de mayor calidad y en la propia evaluación de la experiencia.

\section{CONCLUSIONES}

Las principales conclusiones de la experiencia desarrollada en esta comunicación son las siguientes:

- A raíz de la convocatoria de los Grupos de Innovación Educativa de la UDC se ha conformado un grupo de trabajo que ha implantado de forma coordinada metodologías activas para abordar los contenidos relacionados con el drenaje urbano en asignaturas de los dos masters oficiales impartidos en la Escuela de Caminos de A Coruña.

- Se ha reconfigurado la docencia de esta temática incluyendo visitas a instalaciones experimentales, observación de ensayos de laboratorio, presentación de trabajos de investigación actuales y realización de modelos numéricos con software profesional.

- La combinación de las experiencias en los modelos físicos de laboratorio y los modelos de simulación numérica ha mejorado la comprensión de los procesos y la interpretación física de las principales variables.

- Las actividades realizadas han permitido incrementar el interés del estudiante por el contenido de la materia y sus aplicaciones reales en su futura carrera profesional. El aumento de la motivación de los alumnos y las alumnas permiten incorporar nuevas 
prácticas y estudios de caso en la docencia de las asignaturas. Se trata por tanto de un proceso que se está retroalimentando.

- La experiencia ha sido muy satisfactoria también para los profesores puesto que permite la toma de decisiones conjunta, ahorra trabajo de diseño de la metodología y da seguridad en su aplicación y la evaluación de la docencia con estudiantes de perfiles diferentes.

- Este proyecto coordinado de innovación docente ha servido también como motivación para los profesores que pretenden su mejora continua y el lanzamiento de nuevas aplicaciones de las metodologías activas a sus materias.

\section{REFERENCIAS}

Augas de Galicia. (2009). Instruccións Técnicas para Obras Hidráulicas en Galicia. Serie Saneamento. Santiago de Compostela: Xunta de Galicia.

Rossman, L. A. (2015). Storm Water Management Model User's Manual Version 5.1. Cincinnati: US-Environmental Protection Agency. 